\title{
Research and Simulation of TCM in Simultaneous Wireless Information and Power Transfer System
}

\author{
Cui Tao, Dong Shiwei, Li Jun, Dong Yazhou, and Hu Yanlang
}

\begin{abstract}
The communication and wireless power transfer system can supply energy while guaranteeing the information delivers, and provide technology guarantee for contingency wireless communication be in time and effective. A implement scheme of the communication and wireless power transfer system is introduced in this paper, and the require of the way of coding and modulation in the communication and wireless power transfer system is also analysed; This paper introduces the principle of TCM technology, introduces an application scheme of TCM in the Simultaneous Wireless Information and Power Transfer System, and analyses the scheme by simulation software.
\end{abstract}

Index Terms-The simultaneous wireless information and power transfer system, TCM.

\section{INTRODUCTION}

The wireless power transfer implements non-contact power transfer mainly by the way of electromagnetic inductive (EMI), electric magnetic resonance, $\mathrm{RF}$, microwave, laser etc. Along with the development of electric power and electron implement, power conversion and control technology, the wireless power transfer obtains breakthrough gradually in conversion rate and low radiation etc [1]. The wireless power transfer presents good application foreground in many fields, such as military, communication, industry, medical, transportation, electric power, aviation and spaceflight, energy saving and environmental protection etc. The Simultaneous Wireless Information and Power Transfer System can supply energy while guaranteeing the information delivers, and provide technology guarantee for contingency wireless communication be in time and effective [2].

\section{The SimultaneOUS WIRELESS INFORMATION AND POWER TRANSFER SYSTEM}

The way of wireless power transfer in common has three categories currently, they are electromagnetic inductive, electromagnetism resonance coupling and microwave power transfer. Electromagnetic inductive and electromagnetism resonance coupling cannot implement power transfer in far distance limit to the basic principle, the distance of electromagnetic inductive commonly within $10 \mathrm{~cm}$, and the

Manuscript received March 5, 2015; revised November 4, 2015. This work was supported by The National Foundation of China under Grant No.9140A20090314HT05310.

The authors are with National Key Laboratory of Science and Technology on Space Microwave, Xian 710100, China (e-mail: cuit81@cast504.com, sw.dong@163.com, lijun206@sina.com, yazhoudong@gmail.com, Huyl39@cast504.com). furthest distance of electromagnetism resonance coupling in news is $2 \mathrm{~m}$; Also, due to the low frequency that the two ways adopted, the information rate is limited [3], [4]. Compared to the two ways above, the microwave wireless power transfer can implement power transfer in far distance, and obtain high communication rate at the same time.

The Simultaneous Wireless Information and Power Transfer System this article introduced implement simultaneous transfer with information and power, adopts the microwave wireless power transfer, and uses encoding and microwave power modulation technology. The diagram of this system is shown below.

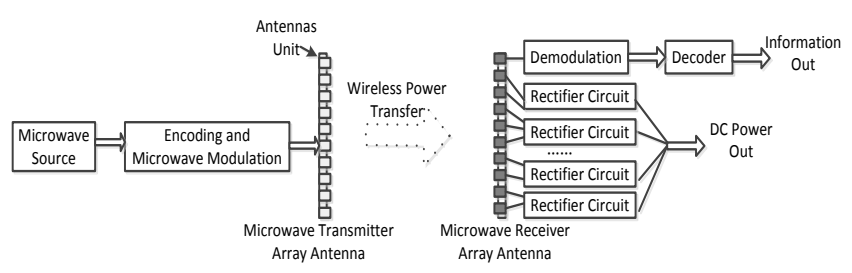

Fig. 1. Diagram of the simultaneous wireless information and power transfer system.

The Simultaneous Wireless Information and Power Transfer System transfers microwave wireless power together with information transformation, guaranteeing the efficiency of microwave power transfer as well as guaranteeing stabilization of characteristic of RF of microwave channel, at the same time needs coding and modulation cannot cause large amplitude jitter of carrier and the spectrum of modulated signal be narrow, so selecting appropriate way of coding and modulation becomes the key of guaranteeing transmission efficiency in the Simultaneous Wireless Information and Power Transfer System.

\section{RESEARCH ON TCM TECHNOLOGY IN THE SIMULTANEOUS WIRELESS INFORMATION AND POWER TRANSFER SYSTEM}

Trellis-coded modulation (TCM) is a combined coding and modulation technique that introduces forward error correction (FEC) coding without increasing the bandwidth of the channel signal. TCM introduces redundant bits through signal set expansion rather than additional transmitted symbols. And one performance comparison that is made is with uncoded systems that have the same spectral efficiency [5]. With TCM the spectral efficiency is defined as the number of information bits per transmitted symbol, and any comparison with uncoded systems is made with those which have the same average energy per transmitted symbol and the same spectral efficiency. TCM resolve problem that obtain obvious coding gain and make the frequency and power efficiency be best in 
the condition of not increasing bandwidth of channel and not reducing information rate, and suitable for the demand of narrowband of the Simultaneous Wireless Information and Power Transfer System [6]. So the Simultaneous Wireless Information and Power Transfer System introduces TCM technology, and implement by $(2,1,3)$ convolutional coding and 8PSK.

\section{A. Principle of the Simultaneous Wireless Information and Power Transfer System}

The transmitting end modulates input data by TCM, then the modulated data becomes baseband signal through digital shaping filter, the baseband signal is modulated by microwave modulation, and is sent by transmitter. The wireless signal is received by receiver, and is microwave modulated, then the baseband signal is modulated and decoded by 8PSK and Viterbi decoding.

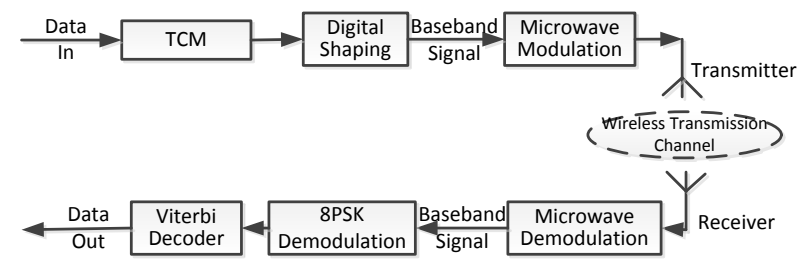

Fig. 2. Principle of the simultaneous wireless information and power transfer system.

\section{B. Research on TCM Technology}

In an environment with power limitations, the communication system must achieve the desired performance with the smallest possible power. Error-correction coding, which adds extra bits to the information bit stream, increases the power efficiency as well as the required bandwidth. In an environment with bandwidth limitations, a high-order modulation scheme such as MPSK increases the spectral efficiency but increases power requirements. TCM combines the operations of modulation and coding in one step and introduces error correction coding without increasing bandwidth [7].

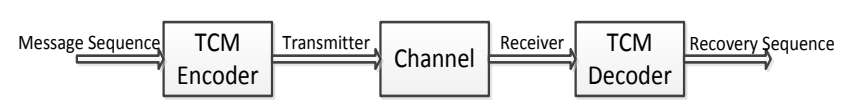

Fig. 3. Digital communication system based on TCM.

In order to design a TCM system three basic steps must be followed. The first step is the appropriate selection of the signal constellation that meets the spectral efficiency specifications. Second is the selection of the code. The last step is to label the signal set in order to maximize the minimum squared-Euclidean distance of the overall TCM system. The technique used to assign these labels is called mapping by set partitioning.

In general, there are $\mathrm{m}$ different TCM implementations. If we apply one of the $\mathrm{m}$ information bits to a convolutional encoder with code rate $r=1 / 2$, then the output of the encoder selects a symbol subset from the second partition level where each symbol subset contains $2^{\wedge}(\mathrm{m}-1)$ symbols. The (m-1) remaining uncoded bits select one of the $2^{\wedge}(\mathrm{m}-1)$ symbols signal from the selected subset. In this case each branch of TCM trellis has $2^{\wedge}(m-1)-1$ parallel transitions. If we apply two information bits to a $r=2 / 3$ convolutional encoder, the output of the encoder selects a symbol subset from the third partition level where each symbol subset contains $2^{\wedge}(m-2)$ symbols. The $(m-2)$ remaining uncoded bits select one of the $2^{\wedge}(m-2)$ symbols signal from the selected subset. In this case each branch of TCM trellis has $2^{\wedge}(m-2)-2$ parallel transitions. If we continue the above process until all $\mathrm{m}$ information bits are applied to an $r=m /(m+1)$ convolutional encoder, then the output of the encoder selects a symbol subset from the $(m+1)$ partition level where each symbol subset contains only one symbol [8]. In this case there are no parallel transitions, which results in no error floor due to parallel transitions, but as $\mathrm{m}$ increases and the code rate increases, so does decoder complexity. Fig. 4 shows a generic structure TCM encoder.

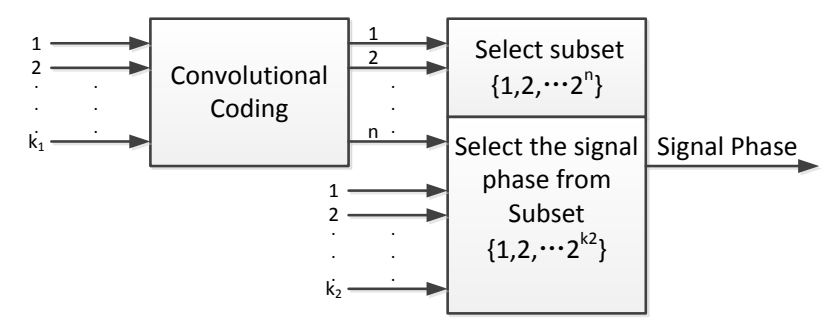

Fig. 4. Set partitioning TCM encoder diagram.

The error trellis of the convolutional code is an alternative graphical representation of the code that is equivalent to the state diagram of the specific code. A well known algorithm for decoding is the Viterbi algorithm (VA), which is a maximum likelihood decoding algorithm and relatively simple to implement. Viterbi algorithm decodes the convolutional code by selecting the most likely path through the trellis which represents the received code sequence and is associated with a given received information sequence.

The key point of TCM is, instead of using redundant bits, signals that represent symbols, where some of the bits represented by the symbol are redundant and some are not, are transmitted. The potential to transmit signals over bandwidth-constrained channels with increased data rates, coding gain, and no bandwidth expansion makes TCM suitable for satellite, mobile, and networking applications.

\section{Implementation of TCM}

Fig. 5 shows a implementation structure of communication transmission system. The Transmitter includes channel encoder and modulator, the signal pass through channel, and the receiver obtain output data via demodulator and decoder, thereby gains better transmission efficiency.

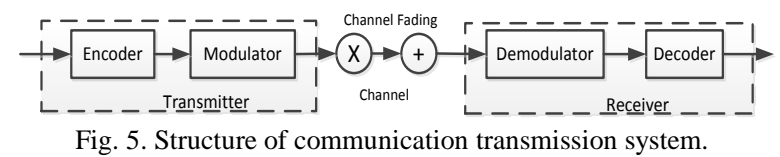

Fig. 6 shows diagram of TCM system. The source firstly input series/parallel conversion, then becomes two-way signal $i$ and $q$; signal $i$ and $q$ pass through channel, the receiver receive signal $I$ and $Q$, then signals input Viterbi decoder and become output information after parallel/series conversion.

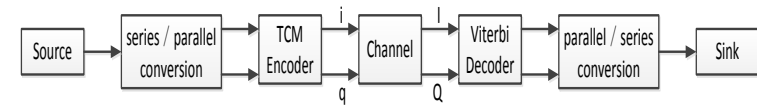

Fig. 6. Diagram of TCM system. 
Fig. 7 shows diagram of convolutional coding.

Coding rate: $r=1 / 2$, constraint length $k=3$.

Coding polynomials:

$$
\begin{gathered}
x_{1 j}=m_{j} \oplus m_{j-1} \oplus m_{j-2} \\
x_{2 j}=m_{j} \oplus m_{j-2}
\end{gathered}
$$

The initialize information of coding polynomials are' 0 '.

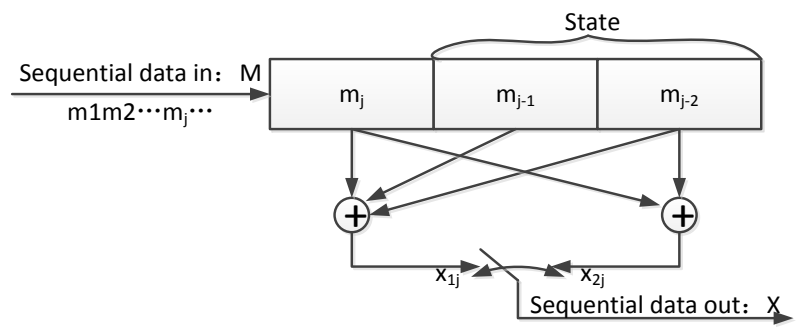

Fig. 7. Diagram of convolutional coding.

Viterbi decoder is the emphasis and nodus of the design. Fig. 7 shows implementation of Viterbi decoder. Viterbi decoder contains three parts: Branch Metric, ACS Part(Path Metric, ACS and Survival Path), Decoder Out.

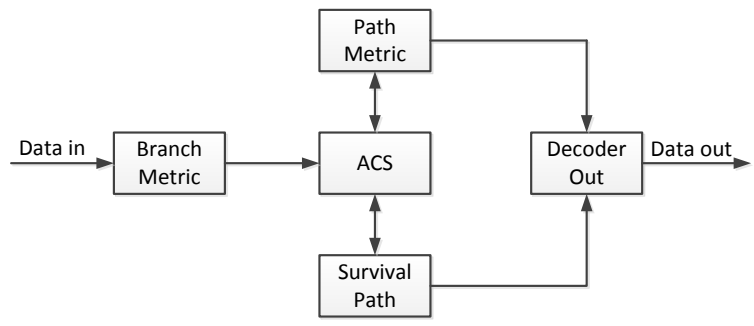

Fig. 8. Implementation diagram of Viterbi decoder.

\section{SimUlation AND ANALYSIS OF TCM IN THE SIMULTANEOUS WIRELESS INFORMATION AND POWER TRANSFER SYSTEM}

The frequency of carrier in the Simultaneous Wireless Information and Power Transfer System is $5.8 \mathrm{Ghz}$, transmit power is $30 \mathrm{~W}$, and the simulation environment of wireless channel is Gauss channel, obtains constellation chart of 8PSK, simulation chart of modulated signal (I, Q), and compare curve chart of between TCM BER and 8PSK theory BER.

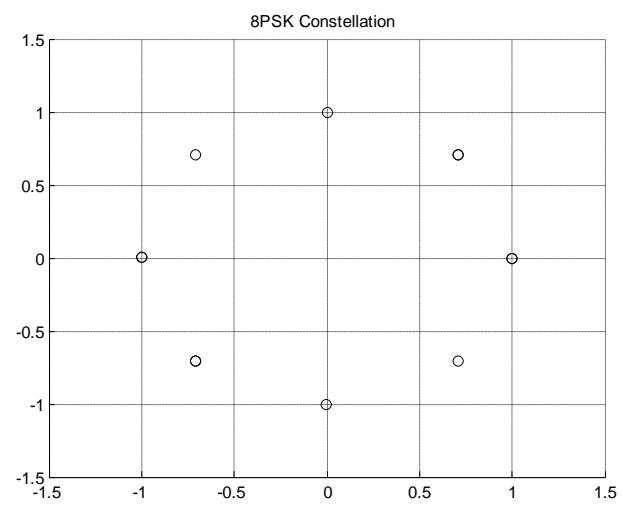

Fig. 9. Constellation chart of 8PSK.
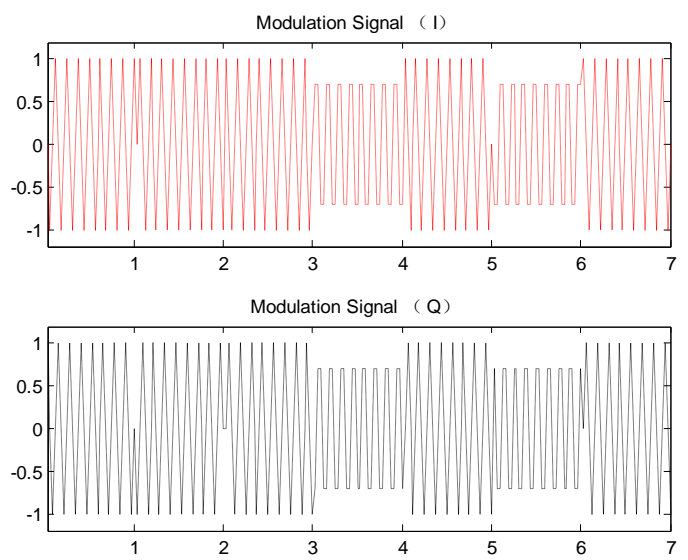

Fig. 10. Simulation chart of modulated signal (I,Q).

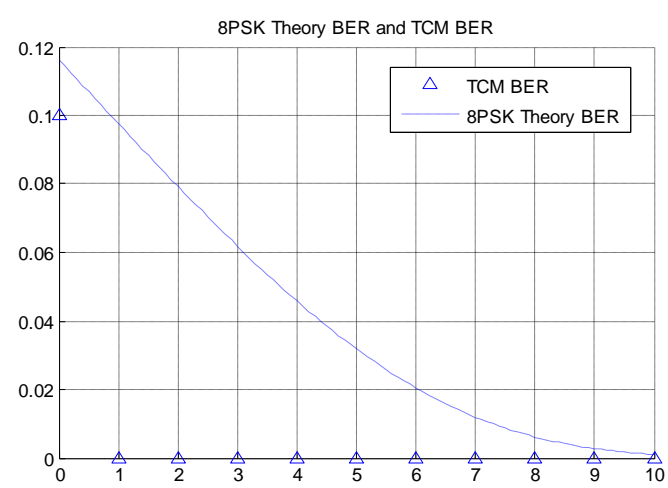

Fig. 11. BER curve of 8PSK and TCM.

Fig. 11 shows BER curve of 8PSK and TCM. From the figure we see that compared to the BER performance of traditional 8PSK, 8PSK TCM can obtain lower BER in the condition of stated SNR. TCM can improve transmission performance of signals under the circs of not increasing bandwidth of channel and not reducing information rate.

\section{CONCLUSIONS}

Trellis-coded modulation (TCM) gets an extensive application in more and more fields, and suitable for the Simultaneous Wireless Information and Power Transfer System which power and spectrum is limited. From the simulation result of TCM scheme, we see that TCM has character of high rate and anti-noise relative to the traditional coding and modulation system. Therefore research improve and optimize the methods of coding and modulation, not only has theoretic meaning on area of coding and modulation, but also makes information transfer develop towards the way of higher rate and lower BER, and certainly have wider foreground in the communication and wireless power transfer area.

\section{ACKNOWLEDGMENT}

The research work was supported by the National Foundation of China under Grant No.9140A20090314HT05310.

\section{REFERENCES}

[1] S. Riviere, A. Douyere, F. Alicalapa, and J. D. L. S. Luk, "Study of complete WPT system for WSN applications at low power level," Electronics Letters, vol. 46, no. 8, pp. 597-598, April 15, 2010. 
[2] T. Paing, E. Falkenstein, R. Zane, and Z. Popovic, "Custom ic for ultra-low power rf energy harvesting," IEEE APEC 2009, pp $1239-1245,2009$.

[3] S. A. Bhalerao, A. V. Chaudhary, R. B. Deshmukh, and R. M. Patrikar, "Powering wireless sensor nodes using ambient rf energy," IEEE SMC '06, pp. 2695-2700, 2006

[4] M. Philipose, J. R. Smith, B. Jiang, A. Mamishev, S. Roy, and K. Sundara-Rajan, "Battery-free wireless identification and sensing," IEEE Pervasive Computing, vol. 4, no. 1, pp. 37-45, 2005.

[5] K. Pyloudis, "Low spectral efficiency trellis coded modulation systems," M.S. thesis, Naval Postgraduate School, Monterey, CA, 2006.

[6] G. Ungerboeck, "Trellis-coded modulation with reduntant signal sets. Part i: introduction," IEEE Commun. Mag., vol. 25, pp. 5-11, Feb. 1987.

[7] J. G. Proakis, Digital Communications, 4th edition, New York, NY: McGraw Hill, 2001.
[8] I. Katzourakis, "Performance analysis of a variable data rate TCM waveform transmitted over a channel with AWGN and pulse-noise interference," M.S. thesis, Naval Postgraduate School, Monterey, CA, 2007.

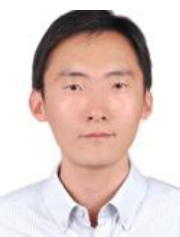

Cui Tao was born on September 10, 1984, Shandong province, China. He graduated in the major of communication and information system with the master degree in 2011 from Xidian university, Xi'An, Shaanxi province, China. He works on the communication signal processing and attends the national projects on switching and signal processing. 